\title{
From Giza to the Pantheon: astronomy as a key to the architectural projects of the ancient past
}

\author{
Giulio Magli \\ Faculty of Civil Architecture, Politecnico di Milano \\ Piazza Leonardo da Vinci 32, 20133 Milano, Italy \\ email: Giulio.Magli@polimi.it
}

\begin{abstract}
In many of the "wonders" of our past, information about their meaning and scope has been encoded in the form of astronomical alignments to celestial bodies. Therefore, in many cases, understanding the ideas of the ancient architects turns out to be connected with the study of the relationship of their cultures with the sky. This is the aim of archaeoastronomy, a discipline which is a quite efficacious tool in unraveling the original projects of many monuments. This issue is briefly discussed here by means of three examples taken from three completely different cultures and historical periods: the so-called "air shafts" of the Great Pyramid, the urban layout of the capital of the Incas, and the design of the Pantheon.
\end{abstract}

Keywords. Archaeoastronomy, Egypt, pyramids, Inca architecture, Cusco, Pantheon

\section{Introduction}

Most of the "wonders" of our ancient past have passed on to us without being accompanied by written information about their scope, meaning and project. This is obviously the case for monuments whose builders did not - as far as we know- have written language, such as the megalithic temples of Malta or the Inca citadel of Machu Picchu. However, this is also the case of many magnificent monuments which were built -for instance- by the Egyptians and the Romans. Just to mention two cases, about the Giza pyramids and the Pantheon, existing sources are quite vague and/or too late to be fully readable. As a matter of fact, however, there is no doubt about the interest of the builders for the celestial cycles. Witness are in a sense the stones themselves, since astronomy was incorporated as a key ingredient of the building projects. Therefore, in many cases understanding the ideas of the architects of the past, as well as their religious and symbolic world, turns out to be connected with the study of their relationship with the sky, i.e., of Archaeoastronomy. In recent years this discipline -meant in the broader sense of the study the ancient "built" landscape in relation with the sky at the time of construction- has been able to free itself from many existing prejudices on the side of human sciences, as well as getting rid of that noisy background of "fringe" theories which biased its development for many years. The result is a powerful scientific tool which helps us to understand the thought, religion and science of our predecessors (for a complete introduction see Ruggles 1999; Aveni 2001; Magli 2009a).

The aim of the present paper is to briefly illustrate three examples in which Archaeoastronomy has proven to be a powerful instrument of investigation in the "reverse design" analysis of some of the wonders of our past. 


\section{The so-called air shafts of the Great Pyramid}

The architectural complexes of the three main pyramids of Giza and their annexes were constructed in the course of a relatively short period of time (between 2550 and 2480 BCE circa) as tombs for the pharaohs Khufu, Khafre and Menkaure of the 4th Egyptian Dynasty (see Lehner (1999) for a complete, up-to-date introduction to these monuments). The Giza pyramids, together with their temples, are, still today, among the most remarkable architectural achievements of the whole human history. Although writing existed in Egypt since many centuries, from the period of the 4th dynasty no written source is available documenting the planning and the construction procedures of these monuments. However, there can be doubt that astronomy played a fundamental rôle in their planning. It is indeed known since Petrie (1883)'s survey that the sidebases are oriented to the cardinal points with astonishing accuracy. This fact raises a series of interesting issues (for a complete overview on "Pyramids and Stars" see Magli and Belmonte (2009), and references therein). As a key example I will focus here on a architectural element of the Khufu pyramid which -although it may seem incredibleremained something of a mystery up to relatively recent years (actually, still today some scholars tenaciously refuse the fact that Archaeoastronomy has proven to be the key for its interpretation). I am speaking, of course, about the four narrow shafts which cross diagonally the body of the pyramid starting from the North and South walls of the King and Queen's chamber, respectively. These shafts were carefully constructed layer after layer and therefore during the course of many years. The upper shafts, those of the so-called King's chamber, exit on the outer northern and southern faces of the pyramid, respectively, approximately at the same height, while the lower ones, those departing from the so-called Queen's chamber, end at a certain (similar) height with two carefully constructed sliding doors endowed with copper handles, discovered in the course of explorations carried out first by Rudolph Gantenbrinck and after by Zahi Hawass and the National Geographic Society.

No doubt, the builders must have had a quite important and serious motivation, to carry out the construction of these shafts. Nevertheless, it had been usually thought in the past -and can be found in guides and scholarly books still today- that the channels were constructed to assure the ventilation of the chamber during its construction, a theory which is blatantly illogical for several reasons (for instance, a single, vertical shaft running alongside a wall would have done a much better job). Actually, the (astronomical) solution to this interesting enigma -at least for the upper channels - has already been found in 1964, a period in which archaeoastronomy was not yet "born". Perhaps for this reason it remained practically unnoticed up to the huge debate stimulated by the exploration of the lower shafts which occurred in the 90s. Anyhow, in 1964, Egyptologist Alexander Badawy and astronomer Virginia Trimble already had the idea of shifting the sky back in time, observing it as it would have been seen in Giza during the age of the pyramids. They soon realized that, during the years in which Khufu's pyramid was certainly built, say sometime between 2600 and 2450 BCE, the northern shaft of the King's Chamber pointed towards the culmination of the "pole star" of the time, $\alpha$ Draco, while the southern shaft pointed towards the culmination of Orion's Belt (Trimble 1964; Badawy 1964). A similar interpretation holds true for the Queen's chamber shafts, which point an area near the celestial north pole, probably towards culmination of the star Kochab, and to the culmination of Sirius respectively (Bauval 1993).

The reason which led to the construction of the shafts and to the choice of their "targets" can be found in the Pyramid Texts. These are funerary texts which were written in the chambers of later pyramids. They describe the destiny of the deceased pharaoh 


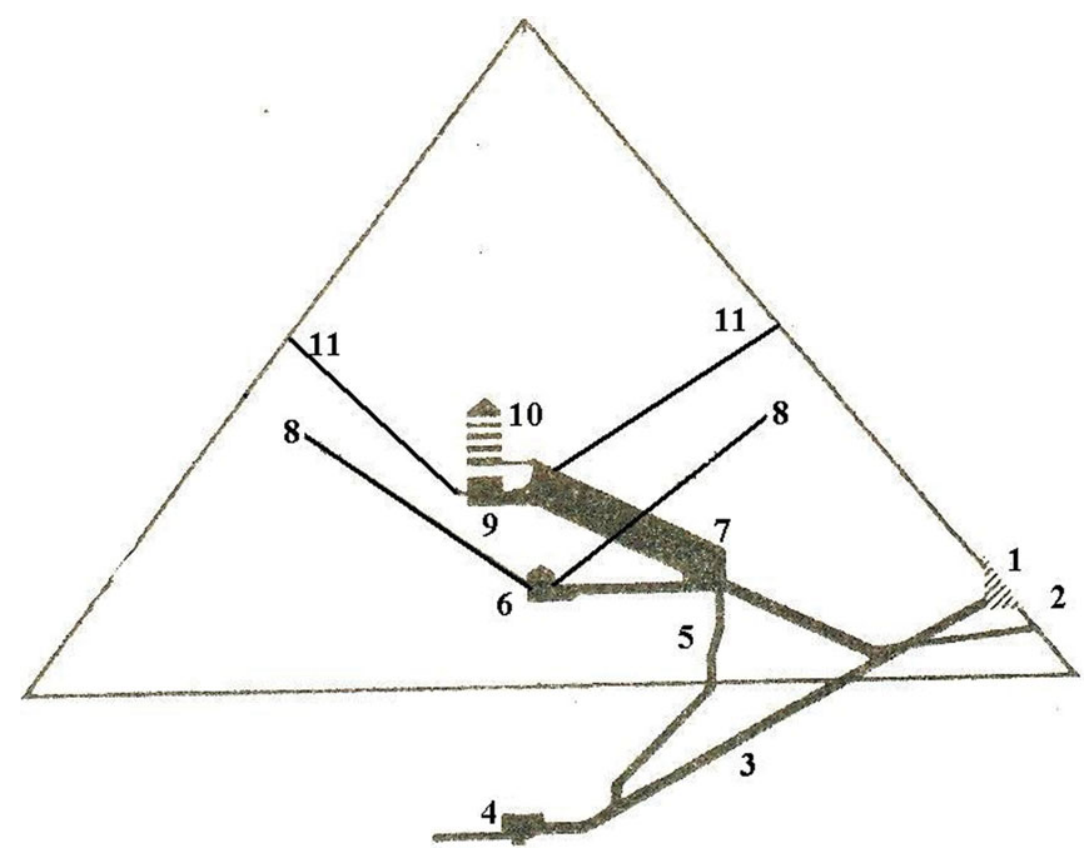

Figure 1. North-south section of the Great Pyramid. (1) Entrance; (2) Forced entrance; (3) Descending corridor; (4) Subterranean Chamber; (5) Well; (6) Queen's Chamber; (7) Great Gallery; (8) Lower shafts; (9) King's chamber; (10) "Relieving Chambers"; (11) Upper shafts.

into two "regions of rebirth", one to the North, together with the "imperishable" (i.e. circumpolar) stars, another "to the South" in the Sirius-Orion region. The four shafts had thus been created with only one purpose in mind, guiding the deceased to the rebirth regions of the sky; of course, it is important to keep in mind that the shafts start off with an horizontal section. Further, their slopes exhibit a few slight changes of angle due to the necessity of avoiding structural elements, before becoming regular in proximity of the exits. So, it would have been impossible to frame celestial bodies in the channels: their alignments had a purely symbolic significance.

The stellar interpretation of the four shafts in Khufu's pyramid is - at least in my view - a shining example of how archaeoastronomy can provide us with a key to understanding some great riddles of the past. It helps, actually, in solving another unanswered riddle about the internal design of the pyramid namely, why the King's Chamber was not built on the vertical from the apex (as was instead customary for the sarcophagus chamber). In fact, it is not possible to reconcile these three requirements:

(a) the stellar, hence fixed, orientation of the shafts;

(b) the exit of the shafts at the same height;

(c) the chamber set below the apex.

Apparently, the will of putting the exits of the shafts at the same height was held to "privileged", compared to the "traditional" arrangement of the sepulchral chamber on the axis.

\section{Cusco and the "dark constellations" of the Incas}

The earliest information regarding the Incas date back to 1200 . They were a bellicose tribe native of the Cusco area who, in less than two centuries, managed to build a very 
large empire, including the south-western American territories which today span between Colombia and Argentina.

The Incas claimed for a direct lineage from the Sun; however their religious feelings included a variety of "holy things". In a certain way the entire landscape, with its places (huaca) directions (ceque) and mountains (apu), was considered sacred. Beyond the level of the sacred landscape there was a superior level -the celestial one- and an inferior one -that pertaining to the Earth; these three levels were connected by the flowing of the rivers from Earth into the great celestial river, the Milky Way or Mayu.

The Inca state - called Tahuantinsuyu or "The Four Parts of the Earth" - was organised according to a centralised framework and was managed thanks to the use of a common language. The state bureaucracy kept an accurate account of the population and economic administration; the data were stored in quipus, clusters of ropes tightened to a main one and laid out as a "tree". Despite the existence of various clues of their existence, there no known surviving quipu carrying written texts, and therefore the only written information we have on architectural, religious and symbolic ideas of the Incas is that reported on the historical chronicles of the Spanish conquest, which are, of course, highly distorted by the viewpoint of the conquerors. Further, some spectacular architectural achievements of the Incas - such as the towns of Pisac and Machu Picchu- were not even cited in them. All in all, at present, all the monuments and the towns built by the Incas remain essentially silent. I shall briefly discuss a case study in which I have recently proposed to use astronomy as a key to unravel at least part of the symbolism which was endowed in Inca town planning (Magli 2005).

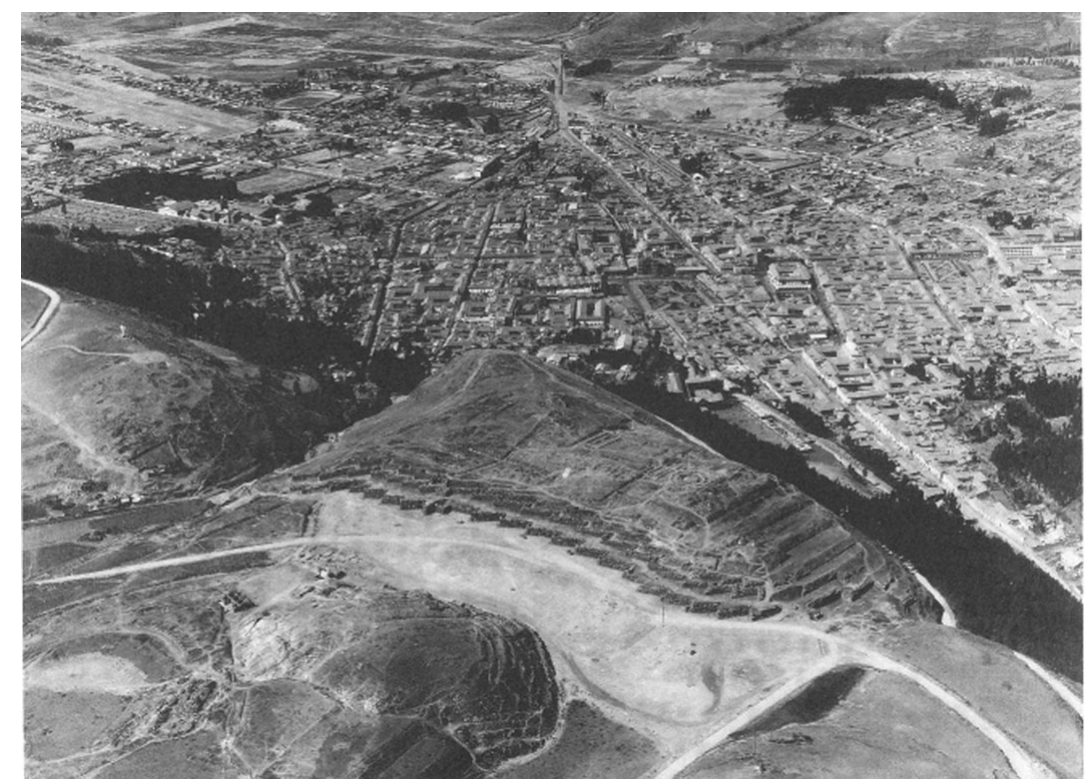

Figure 2. A old air photograph of Cusco from the North-West. The huge hill of the Sacsahuaman is in the foreground, while the "tail" of the Puma is clearly visible in the background, outlined by the two converging roads.

The problem regards the shape of the capital, Cusco. Cusco was a splendor, unbelievably rich in works of art and of extraordinary architectural constructions. The original plan of the Inca city is still perfectly discernible today, since the Spaniards built their residences over the Inca palaces. The town was planned at the confluence between two rivers. 
There was no conceivable practical reason for doing this strange choice: the town could have been built in the plain some hundred meters to the South without any problem. As a consequence of the "crazy" layout, the streets cross longitudinally at different angles and the blocks of buildings (most of which still exhibit the Inca marvelous stonework on their foundations) are not rectangular but trapezoidal, because of the need to adjust the layout within to the two converging rivers (today covered by roads). Why?

The chronicler Sarmiento de Gamboa, in his work written in 1572, says (transl. by Clements Markham, 1907) that the great Inca king Pachacuti "had called the city of Cuzco the lion city. He said that the tail was where the two rivers unite which flow through it, that the body was the great square and the houses round it, and that the head was wanting". As a consequence, Pachacuti's son decided that "the best head would be to make a fortress on a high plateau to the North of the city" and so the famous "fortress" called Sacsahuaman was constructed on the hill. Cusco is therefore a unique case in the world (as far as I know, at least) of a town built after a plan conceived to satisfy some purely symbolic criteria (this monumental re-assessment of the city happened probably during the first half of the 15 th century, over a pre-existing site; the conventional date for this event is fixed at 1438). It is actually easy to see the profile of the animal still today when looking at a map of the historical centre of Cusco: the main square is the space between the front and the back legs of the animal, while the main temple, the Temple of the Sun or Coricancha, correspond to its genitalia (Gasparini \& Margolies 1980). Following the contour of the animal, we see that the head is really represented by the hill, called Sacsahuaman, located at the North-West end of the town plan. At the northern end of the hill are the magnificent zig-zag megalithic terraces which render the Sacsahuaman one of the masterpieces of Inca architecture.

In front of such a huge and complex urban project, it is natural to ask for its motivations. This problem is unsolved, to the point that some scholar has refused the interpretation of the town's layout in terms of the Puma shape, proposing that the parallel with the feline was only a metaphor of the centralized power (Zuidema 1983). Actually however, the Puma was certainly a sacred animal . The iconography of the Puma recursively appears in the few remaining sculptures of Inca time (for instance, on the Sayhuite stone, in the ceremonial sector of Huanuco Pampa, and on recently found vessels in the Kenko area of Cusco) as well as in immediately post-conquest reliefs in Cusco (Trever 2007). The same holds for the serpent iconography and the two animals often appear together, such as, for instance, in various Inca reliefs and in the "Inca coat of arm" depicted by the chronicler Poma de Ayala. Now it is well known that a key characteristic of Andean religion and way of thinking was the existence of dual, complimentary aspects, such as, for instance, the fundamental duality between hanan (above) and hurin (below). Taking into account this fact, I have proposed that the Puma layout of Cusco and the characteristic serpent-like zig-zag shape of the Sacsahuaman might have been connected with "duals" in the sky in the form of two dark cloud constellations.

The dark nebula, or dark clouds, constellations are a way of representing regions of the Milky Way which is alien to western astronomy. The Incas perceived the dark zones inside our galaxy as "figures", actually animal's shapes, and organised the figures into a band of dark constellations which crosses the sky in a way which is in a sense similar to that of our zodiac (Urton 1978, 1982). Among those Inca dark constellations which are securely individuated appear the Fox, the Llama and the Serpent, all located in the "southern" part of the Milky Way. A dark ("crying" i.e. associated to rain) feline however appears in the "cosmological" diagram depicted by the noble Indio Pachacuti Yamqui (1613). This figure might be identified with a dark constellation playing a rôle "dual" to that of the serpent and located at "the other end" of the dark zone inside the Galaxy. 
If this is true then the Puma would be located near Cygnus, where the two branches of the Milky Way converge like do the rivers of Cusco near the Puma's "tail".

A similar "astronomically-related" interpretation can be developed for the Inca town of Machu Picchu. The location of this town might in fact have been chosen to act as the ideal counterpart of the Inca pilgrimage center of the Island of the Sun on the Titicaca lake (for details see Magli 2009b).

\section{The Pantheon}

The Pantheon is the best preserved monument of the Roman period in Rome. Originally built by Agrippa under Augustus' rule, it was rebuilt during Hadrian's reign, around 126 BCE. Its elegant and simple architecture is based on a huge hemispherical dome (43.3 metres of diameter) built over a cylinder of the same diameter, as high as the radius. Therefore, the ideal completion of the upper hemisphere touches the central point of the roof, directly under the unique source of natural light of the building. It is the so called oculus, a circular opening on the top of the cupola. No direct sunlight can enter from the door in the course of the whole year, since the gates opens to the North (more precisely, $5^{\circ}$ West of North); as a consequence, the huge building, with its granite columns standing in the pronao, looks as "cold". From the exterior, it usually (that is, for most part of the year) gives to the visitor a strange impression of coldness and darkness, contrary to what happens, for instance, with the Greek temples, which are usually orientated within the arc of the rising sun. Interestingly, the Pantheon can be put together to the Giza pyramids in being a monument of a fully literate culture about which however practically no written information remains. Indeed, only two Roman sources mention the pantheon: Pliny, who just reports about two statues in it, and the historian Cassio Dione, writing some 70 years after his construction, who says the following sibylline statement "Perhaps it has this name because, among the statues which embellished it, there were those of many gods, including Mars and Venus; but my own opinion on the origin of the name is that, because of its vaulted roof, it actually resembles the heavens" (Cass. D. 53.27.2, transl. by the author). That's all. We actually do not know why the Pantheon was built and how it was used. There is no doubt, however, that everyone who have visited the Pantheon in a sunny day has got the impression that the circular sunlight beam "wandering" inside it is a key ingredient in understanding its meaning and, more generally, that solar astronomy played a relevant rôle in the symbolic world of the Romans (Hannah 2005; Magli 2008). For a complete discussion of the archaeoastronomy of the Pantheon the reader is referred to Hannah \& Magli (2009), where also the connection with other monuments in the area is discussed in detail. Here, I shall address briefly only the most clear and relevant "cosmological" connection of the Pantheon with the cycle of the Sun.

Since the door opens towards the North, the sunlight beam at noon hits the entrance wall. Let us consider the position of the beam at noon on the wall in the course of the year. At the autumn equinox, it touches the timber of the upper hemisphere. Then it moves up to a maximum, high over the entrance, at winter solstice. Then, it moves down, touching again the timber at the spring equinox. In the subsequent days, the beam moves down illuminating from inside the entrance, which is fully "crossed" around April 21; after, the beam starts moving on the roof towards the center of the building (which of course is not reached since the Sun does not cross the zenith at Rome latitude). From the summer solstice the beam "turns back", re-crossing the entrance between the end of August and the autumn equinox.

We thus see two clear "moments" put in evidence by the architecture of the Pantheon: the spring equinox and the 21 of April. The equinoxes were related to the "apotheosized" 


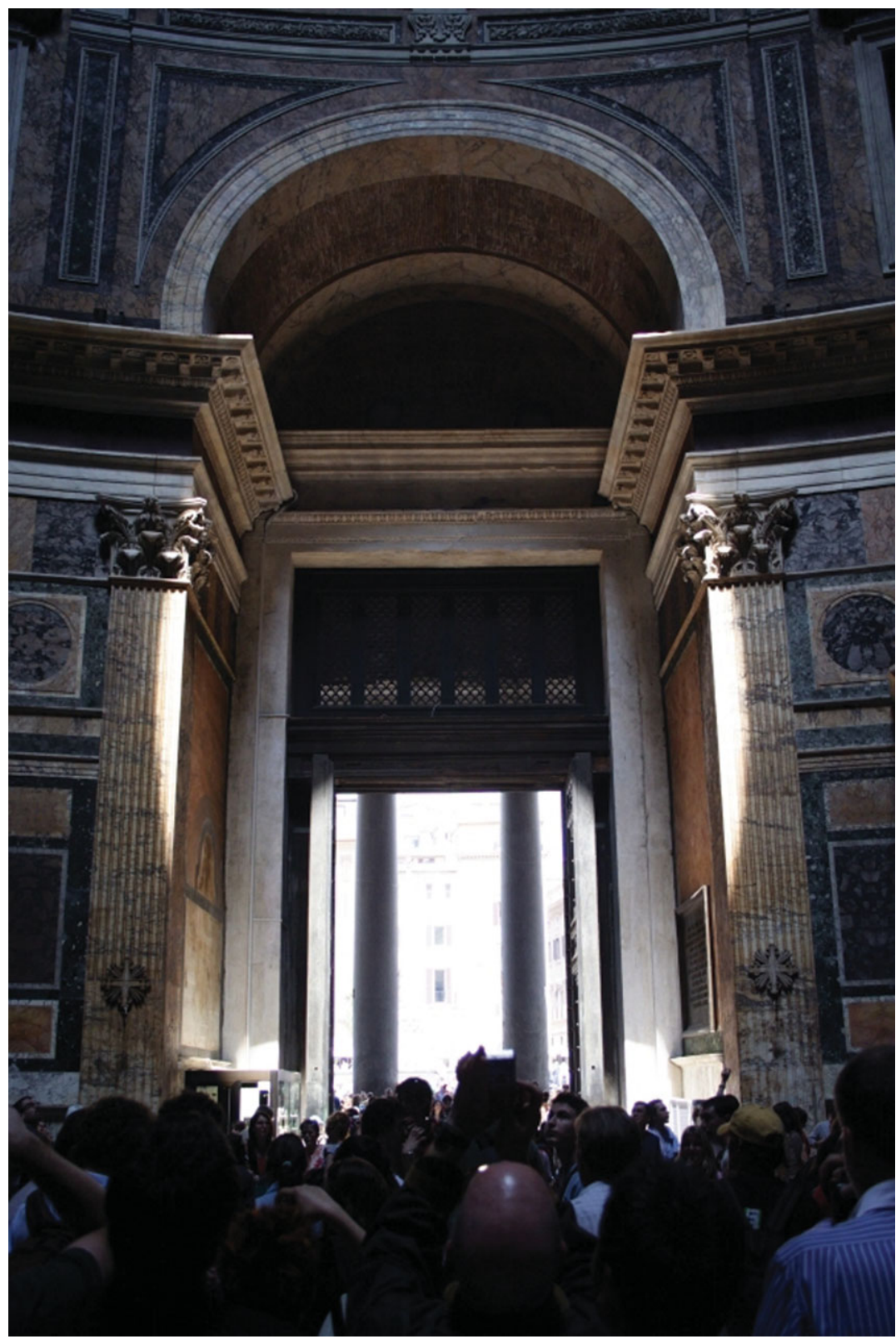

Figure 3. Entrance of the Pantheon, seen from inside at noon on April 21.

emperor, as shown also by a similar Hierophany occurring in Nero's Domus Aurea (Oudet 1992) while the month of April was traditionally devoted to Venus, the Goddess from which the Gens Julia claimed for a direct lineage; the 21 of April is, of course, the traditional date of the foundation of Rome.

All in all, it can be said that the Pantheon, which can be viewed as a giant, symbolic "internal light" sundial (Hannah 2009) manifests to the outside the presence of the Sun inside -first from the fence over the doors, then from the entrance when open- only in this special period of the year, closely related to the symbolic and religious world of 
the Romans (it remains to be ascertained if the symmetric period was related to cosmic significance as well).

\section{References}

Aveni, A. F. 2001, Skywatchers: A Revised and Updated Version of Skywatchers of Ancient Mexico (Austin: University of Texas Press)

Badawy, A. 1964, Mitteilungen des Instituts für Orientforschung, Deutschen Akademie der Wissenschaften zu Berlin, 10, 189

Bauer, B. 1998, The Sacred Landscape of the Inca: The Cusco Ceque System (Austin: University of Texas Press)

Bauval, R. 1993, Discuss. Egyptology, 26, 5

Gasparini, G. \& Margolies, L. 1980, Inca Architecture (Bloomington: Indiana University Press)

Hannah, R. 2005, in XXVIth Annual Meeting of the Australasian Society for Classical Studies Meeting (Dunedin: University of Otago)

Hannah, R. 2009, Time in Antiquity (New York: Rutledge)

Hannah, R. \& Magli, G. 2009, The rôle of the Sun in the Pantheon's design and eaning, http: //arxiv.org/abs/0910.0128

Lehner, M. 1999, The complete pyramids (London: Thames and Hudson)

Magli, G. 2005, Architecture And Mathematics, 7, 22

Magli, G. 2008, Oxford Journal of Archaeology, 27, 63

Magli, G. 2009a, Mysteries and Discoveries of Archaeoastronomy (Berlin: Springer-Verlag)

Magli, G. 2009b, At the other end of the sun's path. A new interpretation of Machu Picchu http://arxiv.org/abs/0904.4882

Magli, G. \& Belmonte, J.A. 2009, in In Search Of Cosmic Order. Selected Essays on Egyptian Archaeoastronomy, J.A. Belmonte and M. Shaltout (eds), (Cairo: American University in Cairo Press), p. 305

Oudet J. F. 1992, Readings in Archaeoastronomy,S. Iwaniszewski (ed) (Warsaw: State Archaeological Museum), p. 25

Petrie, F. 1883, The pyramids and temples of Gizeh (London: Field \& Tuer)

Rowe, J. 1990, Histórica (Lima), 14, 139

Ruggles, C. L. N. 1999, Astronomy In Prehistoric Britain And Ireland (New Haven: Yale University Press)

Trever, L. 2007, Slithering Serpents and the Afterlives of Stones: The Role of Ornament in Inka-Style Architecture of Cusco, Peru, M.A. thesis, University of Maryland

Trimble, V. 1964, Mitteilungen des Instituts für Orientforschung, Deutschen Akademie der Wissenschaften zu Berlin, 10, 183

Urton, G. 1978, Ethnology, 17, 157

Urton, G. 1982, At the Crossroads of the Earth and the Sky: An Andean Cosmology (Austin: University of Texas Press)

Zuidema, T. 1983, J. Lat. Am. Lore 9, 39 